\title{
Bioinformatics and survival analysis of glia maturation factor- $\gamma$ in pan-cancers
}

\author{
Aihua Lan ${ }^{1 \dagger}$, Chunxia Ren ${ }^{2 \dagger}$, Xiaoling Wang ${ }^{2 \dagger}$, Guoqing Tong ${ }^{2^{*}}$ and Gong Yang ${ }^{1,3^{*}}$
}

\begin{abstract}
Background: Glia maturation factor- $\gamma(G M F G)$ is reported to inhibit the actin nucleation through binding to the actin-related protein-2/3 complex (Arp2/3). Considering the main function of GMFG in actin remodeling, which is vital for immune response, angiogenesis, cell division and motility, GMFG is supposed to have important roles in tumor development, while up to now, only two studies described the role of GMFG in cancers. By investigating the clinical values of GMFG using The Cancer Genome Atlas (TCGA) data and the functional mechanisms of GMFG through analyses of Gene Ontology (GO) and Kyoto Encyclopedia of Genes and Genomes (KEGG) pathway enrichments, this study was aimed to better understand the impact of GMFG in pan-cancers and to draw more attentions for the future research of GMFG.

Methods: RNA-seq and clinical data of cancer patients were collected from TCGA and analyzed by the KaplanMeier methods. GO and KEGG analyses were conducted using the online tools from the Database for Annotation, Visualization and Integrated Discovery (DAVID).

Results: Compared to the corresponding normal samples, GMFG was significantly upregulated in glioblastoma (GBM), kidney clear cell carcinoma (KIRC), lower grade glioma (LGG), acute myeloid leukemia (LAML), and pancreatic cancer (PAAD), testicular cancer (TGCT), but was downregulated in kidney chromophobe (KICH), lung adenocarcinoma (LUAD) and lung squamous cell carcinoma (LUSC) $(P<0.05$ for all). High expression of GMFG predicted worse OS in GBM (HR=1.5, $P=0.017)$, LGG $(H R=2.2, P<0.001)$, LUSC (HR=1.4, $P=0.022)$ and ocular melanomas (UVM) (HR=7, P<0.001), as well as worse DFS in LGG (HR=1.8, P<0.001) and prostate cancer (PRAD) $(H R=1.9, P=0.004)$. In contrast, high expression of GMFG was associated with better OS in skin cutaneous melanoma (SKCM) $(H R=0.59, P<0.001)$ and thymoma (THYM) $(H R=0.098, P=0.031)$, as well as better DFS in bile duct cancer $(\mathrm{CHOL})(\mathrm{HR}=0.2, P=0.003)$. GMFG was mainly involved in the immune response, protein binding and cytokine-cytokine receptor interaction pathways, and was positively associated with multiple immunomodulators in most cancers.

Conclusion: Our study preliminarily identified that GMFG may cause different survivals for different cancers through modulating tumor progression, immune response status and tissue-specific tumor microenvironment (TME).
\end{abstract}

Keywords: Glia maturation factor- $\gamma$, Cancer, Survival, Bioinformatics, Immune response, Tumor microenvironment

\footnotetext{
* Correspondence: drivftongguoqing@hotmail.com; yanggong@fudan.edu.cn

${ }^{\dagger}$ Aihua Lan, Chunxia Ren and Xiaoling Wang contributed equally to this

work.

${ }^{2}$ Center for Reproductive Medicine, Shuguang Hospital Affiliated to Shanghai University of Traditional Chinese Medicine, Shanghai 200120, China

${ }^{1}$ Central Laboratory, the Fifth People's Hospital of Shanghai, Fudan University, Shanghai 200240, China

Full list of author information is available at the end of the article
}

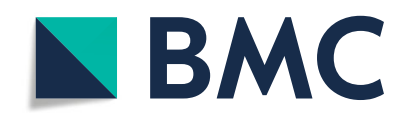

(c) The Author(s). 2021 Open Access This article is licensed under a Creative Commons Attribution 4.0 International License, which permits use, sharing, adaptation, distribution and reproduction in any medium or format, as long as you give appropriate credit to the original author(s) and the source, provide a link to the Creative Commons licence, and indicate if changes were made. The images or other third party material in this article are included in the article's Creative Commons licence, unless indicated otherwise in a credit line to the material. If material is not included in the article's Creative Commons licence and your intended use is not permitted by statutory regulation or exceeds the permitted use, you will need to obtain permission directly from the copyright holder. To view a copy of this licence, visit http://creativecommons.org/licenses/by/4.0/. The Creative Commons Public Domain Dedication waiver (http://creativecommons.org/publicdomain/zero/1.0/) applies to the data made available in this article, unless otherwise stated in a credit line to the data. 


\section{Introduction}

Glia maturation factor- $\gamma$ (GMFG) is a $17 \mathrm{kDa}$ small protein, and its gene sequence is conserved from yeast to mammalian. GMFG was initially identified as a glia maturation factor that can induce brain cell differentiation $[1,2]$. Later reports found that GMFG can regulate the actin cytoskeleton because it actually belongs to the actin-depolymerizing factor homology (ADF-H) family $[3,4]$. All ADF-H proteins can remodel actin cytoskeleton through binding to either actin and/or actin-related proteins (Arps) [5], and the reorganization of actin cytoskeleton is crucial for immune system function, angiogenesis and cell motility [6]. As a novel regulator of the Arp2/3 complex, GMFG is reported to enhance the angiogenic sprouting of zebrafish [7] and to regulate the migration of airway smooth muscle cell [8-10], as well as to promote the chemotaxis of neutrophils and $\mathrm{T}$ lymphocytes [11, 12].

Considering the main function of GMFG in actin remodeling vital for cancer immunity, angiogenesis, cell division and motility [13, 14], GMFG is supposed to have important roles in tumor development, while up to now, only two studies described the role of GMFG in cancers $[15,16]$. These two studies demonstrated that GMFG could promote the migration and proliferation of ovarian and colorectal cancer cells, and the high expression of GMFG was related to poor survival outcome for ovarian cancer patients. To better understand the potential function of GMFG in pan-cancers and to draw more attention to GMFG for the future cancer research, in this study, we investigated the clinical values of GMFG using The Cancer Genome Atlas (TCGA) data, and analyzed the underlying functional mechanisms of GMFG through conducting Gene Ontology (GO) and Kyoto Encyclopedia of Genes and Genomes (KEGG) pathway enrichments. Furthermore, we also evaluated the impact of GMFG on cancer immunity by analysis of the association between GMFG and immunomodulators.

\section{Materials and methods}

\section{Expression and survival analysis}

We used Gene Expression Profiling Interactive Analysis (GEPIA) (http://gepia.cancer-pku.cn/) to compare the GMFG expression between tumor and normal samples [17]. Tumor samples were matched TCGA and Genotype-Tissue Expression (GTEx) normal samples [18], and $\log 2(\mathrm{TPM}+1)$ was used for log-scale. Moreover, the relationship between GMFG expression and pathological stage was investigated in the GEPIA database. Survival analyses that include overall survival (OS) and disease-free survival (DFS) were also conducted in the GEPIA database. Patients were divided into two groups (high and low expression groups) according to the median expression level of GMFG in cancer samples, and the survival curves were generated by the KaplanMeier methods. The hazard ratio (HR) and $P$-value were also calculated. The $P$ value $<0.05$ indicated statistical significance.

\section{GMFG-associated co-expression genes}

To evaluate the potential functional mechanism of GMFG in cancers, we obtained GMFG-associated coexpression genes from the Multi-Experiment Matrix (MEM) (http://biit.cs.ut.ee/mem) [19] and cBioPortal (http://www.cbioportal.org) databases. In MEM database, the associations between GMFG and other genes were evaluated by the score $/ P$-value. Genes with $P$-value smaller than 0.0001 were selected. Meanwhile, the correlations between GMFG and co-expression genes were calculated by the Pearson's correlation analysis in the cBioPortal database, and genes with correlation coefficient (absolute value) more than 0.5 were selected. Finally, the intersection analysis (Venny 2.1, https:// bioinfogp.cnb.csic.es/tools/venny/) was conducted using the selected co-expression genes from both MEM and cBioPortal to acquire the overlapping genes for further analyses.

\section{Functional and pathway enrichment}

To investigate the functional mechanism of GMFG in pan-cancers, the overlapping co-expression genes from MEM and cBioPortal were used for GO and KEGG pathway [20] enrichment analyses. Firstly, the GO and KEGG analyses for each cancer were conducted in Database for Annotation Visualization and Integrated Discovery (DAVID) v6.8 (https://david.ncifcrf.gov), and GO terms and KEGG pathways with $P$-value $<0.05$ were considered as significant enrichments. Thus, significant GO/ KEGG pathways for 32 different cancers were obtained. Then, Venny 2.1 was used for intersection analysis to acquire the top 20 overlapping GO/KEGG pathways among 32 cancers. Finally, the number of co-expression genes for each pathway in each cancer was shown in heatmap.

\section{GMFG and immunomodulators}

Corrections between GMFG with its immunomodulators co-expression genes among 32 cancers were analyzed. The detailed categories of immunomodulators were obtained from the Immune Landscape of Cancer [21] which divided immunomodulators into seven types: costimulator, co-inhibitor, ligand, receptor, cell adhesion, antigen presentation and other. Heatmap was applied to show the correlation between GMFG and immunomodulators. Heatmaps of this study were generated by R language (https://www.r-project.org), other two packages including ggplot2 (version 3.3.3) and ComplexHeatmap (version 2.6.2) were also used to build heatmaps. 


\section{Results}

\section{Expression of GMFG in 33 different cancers}

Among 33 different cancers samples, GMFG expressions in lymphoid neoplasm diffuse large B-cell lymphoma (DLBC), acute myeloid leukemia (LAML) and thymoma (THYM) were obviously higher than that in other cancer types (Fig. 1a). The expression values of GMFG in 29 different cancers and corresponding normal samples were shown in Fig. 1b, four cancers (mesothelioma (MESO), pheochromocytoma and paraganglioma (PCPG), sarcoma (SARC), and uveal melanoma (UVM)) were excluded from the expression profile analysis due to small or a lack of corresponding normal samples. We found that GMFG was significantly upregulated in glioblastoma (GBM), kidney clear cell carcinoma (KIRC), lower grade glioma (LGG), LAML, and pancreatic cancer (PAAD), testicular cancer (TGCT), but was downregulated in kidney chromophobe ( $\mathrm{KICH})$, lung adenocarcinoma (LUAD) and lung squamous cell carcinoma (LUSC) $(P<0.05$ for all).

\section{Pathological stage and survival analysis}

As shown in Fig. 1c, high expression of GMFG was correlated with early pathological stage in bladder cancer (BLCA) $(P=0.016)$, LUAD $(P=0.015)$, skin cutaneous melanoma $(\mathrm{SKCM}) \quad(P=0.006)$ and thyroid cancer

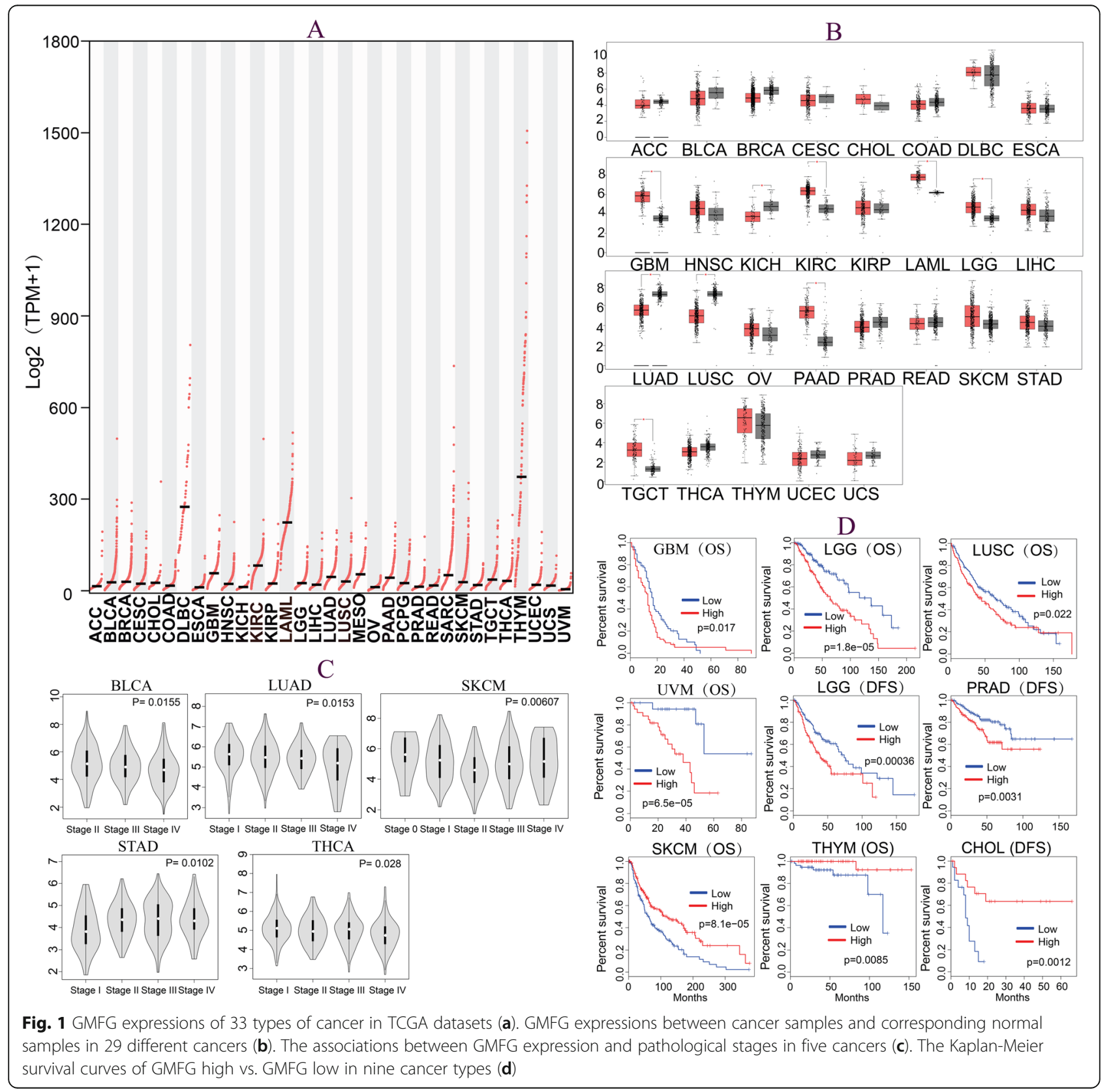


(THCA) $(\mathrm{P}=0.01)$, but was linked with advanced pathological stage in stomach cancer (STAD) $(P=0.028)$. For survival outcome, high expression of GMFG predicted worse $\mathrm{OS}$ in GBM (HR $=1.5, P=0.017)$, LGG $(\mathrm{HR}=2.2$, $P<0.001)$, LUSC (HR $=1.4, P=0.022)$ and UVM $(\mathrm{HR}=$ $7, P<0.001)$, as well as worse DFS in LGG $(\mathrm{HR}=1.8$, $P<0.001)$ and prostate cancer (PRAD) $(\mathrm{HR}=1.9, P=$ $0.004)$. In contrast, high expression of GMFG was associated with better OS in SKCM $(\mathrm{HR}=0.59, P<0.001)$ and THYM $(\mathrm{HR}=0.098, P=0.031)$, as well as better DFS in bile duct cancer (CHOL) $(\mathrm{HR}=0.2, P=0.003)$ (Fig. 1d).

\section{GO and KEGG pathway enrichment analyses}

As demonstrated in Fig. 2a, GMFG was mainly involved in cytokine-cytokine receptor interaction, cell adhesion molecules and chemokine signaling pathways in most cancers except for THYM, PCPG, DLBC, LAML, BLCA, and KIRC. The GO analysis indicated that GMFG was mainly correlated with the biological processes of signal transduction, immune response and inflammatory response in all cancer types with the exception of BLCA, KIRC, LAML and DLBC (Fig. 2b). For cellular component (Fig. 2c), GMFG was mainly enriched in the plasma membrane and integral component of membrane in the majority of 32 cancer types. For molecular function (Fig. 2d), GMFG was strongly related to protein binding in all cancers. Instead of enriching in immune response and plasma membrane, GMFG was linked with the biological processes of translation or insulin receptor signaling pathway, and was mainly concentrated on nucleoplasm or extracellular exosome in THYM, LAML and DLBC (Supplementary file 1-4: Table S1-S4).

\section{Correlation of GMFG and immunomodulators}

The intersective co-expression genes in the top two significant biological processes and KEGG pathways, as well as top one cellular component and molecular function were acquired, and we listed the top 10 overlapping co-expression genes with their corresponding functions in Table 1 . We also summarized the top 10 most significant items of GO/KEGG pathways for each individual cancer (Supplementary file 1-4: Table S1-S4), and we found that GMFG was notably associated with the biological process of immune response in most cancers, so we decided to analyze the correlation between GMFG and its immunomodulators co-expression genes. As shown in Fig. 3, GMFG was positively correlated with immunomodulators genes in most cancer, and the most positive correlations between GMFG and these genes were found in TGCT, SKCM, UVM, LUSC and colon and rectal cancer (COADREAD). As for co-stimulators,

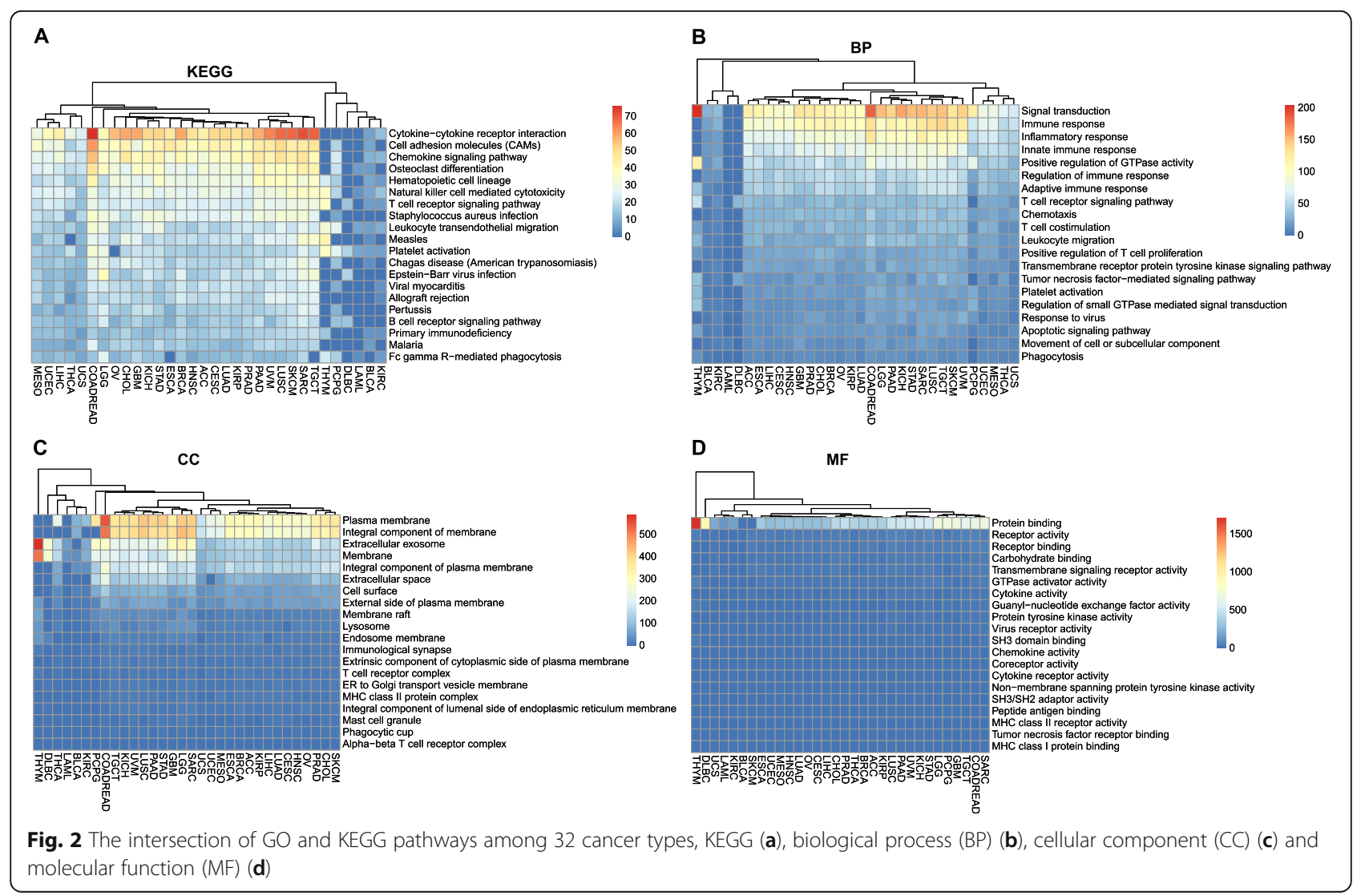


Table 1 The top 10 overlapping co-expression genes and the corresponding functions in the top two significant GO/KEGG pathways

\begin{tabular}{lll}
\hline Category & $\begin{array}{l}\text { Top } \mathbf{1 0} \\
\text { genes }\end{array}$ & Functions \\
\hline $\begin{array}{l}\text { KEGG_PATHWAY hsa04060: Cytokine- } \\
\text { cytokine receptor interaction }\end{array}$ & CSF2RB & $\begin{array}{l}\text { Colony-stimulating factor } \mathbf{2} \text { (granulocyte, macrophage), IL3, IL5 receptor common } \\
\text { subunit beta (high affinity), critical for the activation of both the JAK/STAT (JAK2, } \\
\text { STAT5) and MAP kinase pathways, involved in LYN binding. }\end{array}$ \\
& CCL5 & C-C motif chemokine ligand 5, chemoattractant for blood monocytes, memory T-
\end{tabular}
helper cells and eosinophils, may activate several chemokine receptors including CCR1, CCR3, CCR4 and CCR5.

CXCR3 C-X-C motif chemokine receptor 3, binding CXCL9/Mig, CXCL10/IP10 and CXCL11/ 0.75 I-TAC induce cellular responses that are involved in leukocyte traffic, most notably integrin activation, cytoskeletal changes and chemotactic migration.

LTB Lymphotoxin beta, a type II membrane protein of the TNF family, and an inducer of 0.55 the inflammatory response and involved in normal development of lymphoid tissue.

CD27 A member of the TNF-receptor superfamily, is required for generation and long-term 0.68 maintenance of $\mathrm{T}$ cell immunity. It binds to ligand CD70, and plays a key role in regulating B-cell activation and immunoglobulin synthesis.

Others IL10RA, IL3RA, IL12RB1, CSF1R, CCR5.

KEGG_PATHWAY hsa04514: Cell adhesion molecules (CAMs)

GOTERM_BP_DIRECT GO:0007165 signal transduction
PECAM1 Platelet and endothelial cell adhesion molecule 1, makes up a large portion of endothelial cell intercellular junctions, and might involve in leukocyte migration, angiogenesis, and integrin activation.

CD4 This gene encodes a membrane glycoprotein of T lymphocytes that interacts with MHC II antigens and initiates or augments the early phase of T-cell activation.

CD2 The protein encoded by this gene is a surface antigen found on all peripheral blood T-cells. The encoded protein interacts with LFA3 (CD58) on antigen presenting cells to optimize immune recognition.

ITGB2 Integrin subunit beta 2 , combines with multiple different alpha chains to form different integrin heterodimers. Integrins are cell-surface proteins that participate in cell adhesion as well as cell-surface mediated signaling.

ITGAL Integrin subunit alpha L, combines with ITGB2 to form integrins. 0.73

Others SELPLG, CD86, HLA-DPB1, HLA-DPA1, CD40LG. /

$\begin{array}{ll}\text { PECAM1 As presented above. } & 0.77\end{array}$

RASAL3 RAS Protein Activator Like 3, a Ras GTPase-activating protein, plays an important role in the expansion and functions of natural killer T cells in the liver by negatively regulating RAS activity and the down-stream ERK signaling pathway.

TYROBP TYRO protein tyrosine kinase binding protein, non-covalently associates with activating receptors of the CD300 family. Cross-linking of CD300-TYROBP complexes results in cellular activation, involves in neutrophil activation mediated by integrin.

DOK2 Docking Protein 2 provides a docking platform for the assembly of multimolecular signaling complexes, may modulate the cellular proliferation induced by IL-4, IL-2 and IL-3. DOK2 may involve in modulating Bcr-Abl signaling and inhibit EGF-stimulated MAP kinase activation.

ARHG Rho GTPase Activating Protein 9, a member of the Rho-GAP family of GTPase activatAP9 ing proteins, has substantial GAP activity towards several Rho-family GTPases, converting them to an inactive GDP-bound state. It implicates in regulating adhesion of hematopoietic cells to the extracellular matrix.

Others ARHGAP15, CD4, CD48, CD79B, CD33.

CD4 As presented above. 0.74

CST7 Cystatin-7, a cysteine protease inhibitor with a role in immune regulation through inhibition of the hematopoietic system. Additionally, CST7 promotes metastasis in various cancers.

WAS WASP actin nucleation promoting factor, involves in transduction of signals from receptors on the cell surface to the actin cytoskeleton. WAS associates with the small GTPase, Cdc42 (regulates formation of actin filaments) and the cytoskeletal organizing complex, Arp2/3.

CCL5 As presented above.

VAV1 Vav guanine nucleotide exchange factor 1 , activates pathways leading to actin 
Table 1 The top 10 overlapping co-expression genes and the corresponding functions in the top two significant GO/KEGG pathways (Continued)

\begin{tabular}{|c|c|c|c|}
\hline Category & $\begin{array}{l}\text { Top } 10 \\
\text { genes }\end{array}$ & Functions & Correlation \\
\hline & & $\begin{array}{l}\text { cytoskeletal rearrangements and transcriptional alterations. VAV1 is important in } \\
\text { hematopoiesis, T-cell and B-cell development and activation. }\end{array}$ & \\
\hline & Others & CD79B, LST1, NCF4, S1PR4, LCP2. & / \\
\hline \multirow[t]{6}{*}{$\begin{array}{l}\text { GOTERM_CC_DIRECT GO:0005886 } \\
\text { plasma membrane }\end{array}$} & HCST & $\begin{array}{l}\text { Hematopoietic cell signal transducer, associates with KLRK1 to trigger cytotoxicity } \\
\text { against target cells. KLRK1-HCST receptor plays a role in immune surveillance against } \\
\text { tumors and is required for cytolysis of tumors cells, tumor cells that do not express } \\
\text { HCST escape from immune surveillance mediated by NK cell. }\end{array}$ & 0.86 \\
\hline & TYROBP & As presented above. & 0.91 \\
\hline & PECAM1 & As presented above. & 0.77 \\
\hline & $\mathrm{CD} 2$ & As presented above. & 0.80 \\
\hline & CORO1A & $\begin{array}{l}\text { Coronin } 1 \mathbf{A} \text {, a crucial component of the cytoskeleton of highly motile cells, } \\
\text { functioning in the invagination and protrusions of plasma membrane involved in cell } \\
\text { locomotion. CORO1A involves in a variety of cellular processes, including cell cycle } \\
\text { progression, signal transduction, apoptosis, and gene regulation. }\end{array}$ & 0.71 \\
\hline & Others & CD3D, CD3E, CD79B, GNGT2, KLRB1. & / \\
\hline \multirow{6}{*}{$\begin{array}{l}\text { GOTERM_MF_DIRECT GO:0005515 } \\
\text { protein binding }\end{array}$} & HCST & As presented above. & 0.86 \\
\hline & $\begin{array}{l}\text { TNFA } \\
\text { IP8L2 }\end{array}$ & $\begin{array}{l}\text { TNF alpha-induced protein } \mathbf{8} \text {-like protein } \mathbf{2} \text {, acts as a negative regulator of innate } \\
\text { and adaptive immunity by maintaining immune homeostasis, negatively regulates } \\
\text { Toll-like receptor and T-cell receptor function. TNFAIP8L2 inhibits JUN/AP1 and NF- } \\
\text { kappa-B activation, promotes Fas-induced apoptosis. }\end{array}$ & 0.88 \\
\hline & SPI1 & $\begin{array}{l}\text { Spi-1 proto-oncogene, is a member of the Ets transcription factor family and plays a } \\
\text { vital role in development and maturation of the myeloid and lymphoid lineages. }\end{array}$ & 0.86 \\
\hline & WAS & As presented above. & 0.81 \\
\hline & PECAM1 & As presented above. & 0.77 \\
\hline & Others & LAPTM5, CD37, GPSM3, ARHGAP9, TYROBP. & / \\
\hline
\end{tabular}

GMFG was highly associated with CD80 and CD28 in most cancers. In terms of co-inhibitors, GMFG showed a positive relationship with PDCD1LG2 (PD-L2) and SLAMF7 in most cancers. For cellular ligands, CXCL9, CXCL10, CCL5, CD40LG, IL10 and IFNG had a stronger relationship with GMFG than other ligands. For receptors, TIGIT, PDCD1 (PD-1), CTLA4, IL2RA, TNFR SF4, CD27, LAG3, ICOS, BTLA, ADORA2A and HAVC R2 were highly associated with GMFG in most cancers. As for cell adhesion molecules, GMFG was strongly correlated with ITGB2, followed by ICAM1 and SELP. Regarding MHC molecules, we found that the MHC class II molecules including HLA-DRA, HLA-DRB1, HLA-DPB1 and HLA-DPA1 exhibited a stronger association with GMFG than other MHC molecules. Finally, IDO1 and the cytotoxic molecules including GZMA and PRF1 showed a positively stronger association with GMFG in most cancer types. Interestingly, GMFG showed a significantly positive association with PD-1 and PD-L2, but not with CD274 (PD-L1) in most cancers. In contrast, GMFG was negatively correlated with most of the immunomodulators in THYM, and only a week correlation was observed between GMFG and immunomodulators in DLBC, LAML, and liver cancer (LIHC).

\section{Discussion}

Our study found that, compared with in normal samples, GMFG was increased in six cancers (GBM, KIRC, LGG, LAML, PAAD, and TGCT), but was decreased in three cancers (KICH, LUAD, and LUSC). In addition, high expression of GMFG was associated with early pathological stage in four cancers (BLCA, LUAD, SKCM, and THCA), but was correlated with advanced pathological stage in STAD. Moreover, the associations of GMFG expression with OS and DFS were also investigated, and high expression of GMFG predicted worse OS in four cancers (GBM, LGG, LUSC, and UVM), and worse DFS for LGG and PRAD, but was associated with better OS in SKCM and THYM, better DFS in CHOL. These findings demonstrated significant differences in the expression patterns and prognostic values of GMFG in different cancers, indicating that GMFG may be a valuable biomarker for the diagnosis and prognosis of some cancers. 


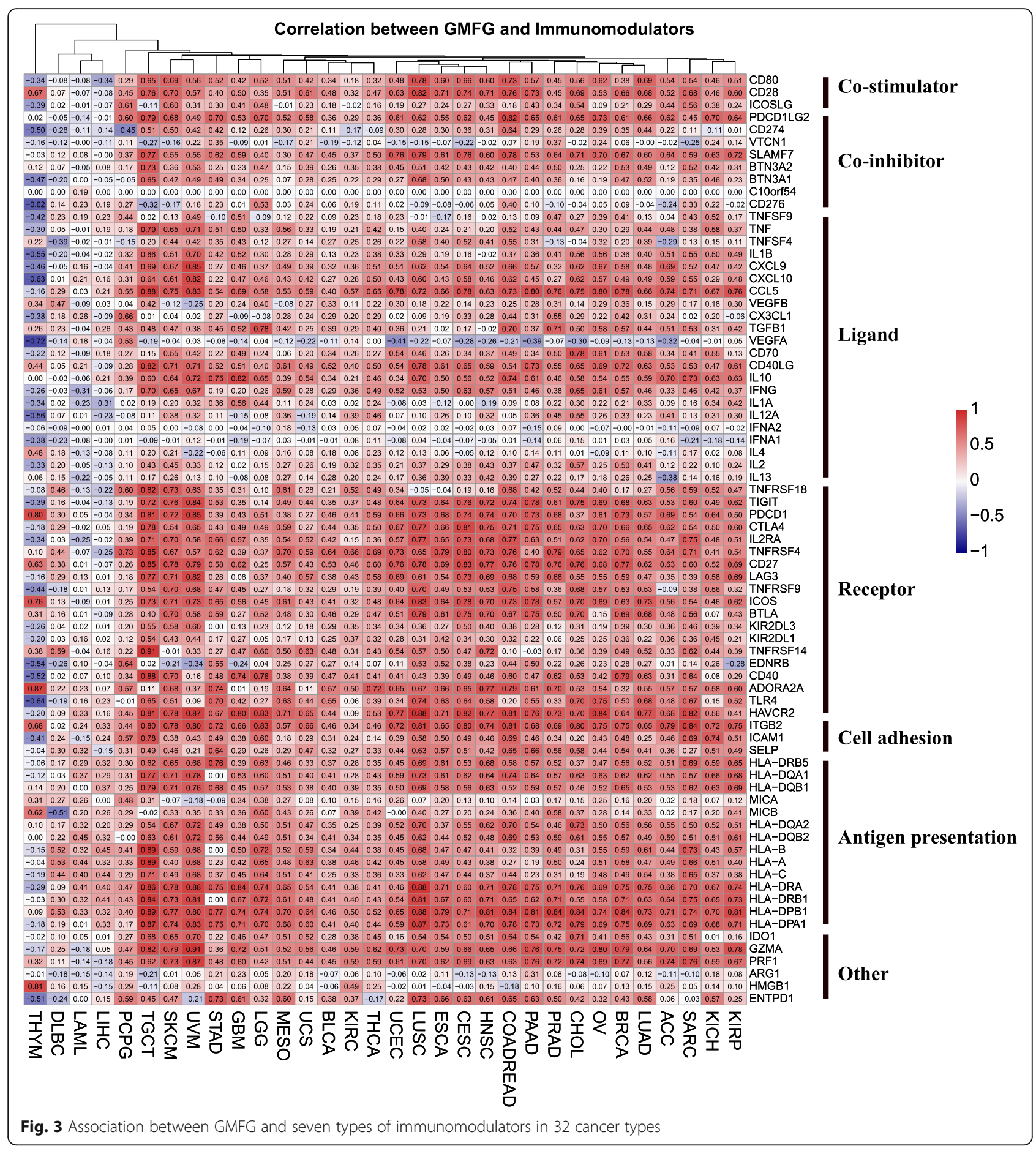

High expression of GMFG predicted different prognoses for different cancers. A possible explanation for this result is that GMFG functions differently in different cancers. First of all, combining the previous reports, we conclude that GMFG may enhance the motility of both cancer cells and immunocytes, because GMFG is mainly involved in protein binding in most cancers according to our study, which is consistent with the fact that GMFG binds to Arp2/3 complex to depolymerize the actin cytoskeleton [13]. In the literature, GMFG promotes the process of angiogenesis [7], and the proliferation and motility of cancer cell through altering the actin cytoskeleton $[15,16]$. Thus, our research data suggest that GMFG plays an important role in tumor development largely through the protein-protein interaction. On the other hand, GMFG is also reported to enhance the 
chemotaxis of neutrophils and $\mathrm{T}$ lymphocytes through remodeling the actin cytoskeleton $[11,12]$. In our study, GMFG is involved in immune response including $\mathrm{T}$ cell receptor signaling pathway, chemotaxis, $\mathrm{T}$ cell costimulation, leukocyte migration and $\mathrm{T}$ cell proliferation regulation, which may also be associated with the function of actin remodeling. Additionally, the biological process of immune response was also found in the KEGG pathways, such as Natural killer cell mediated cytotoxicity and B cell receptor signaling pathway. Taken together, that GMFG enhances both the cancer progression and the immune defense, and patients' survival outcome may depend on the balance between the speed of cancer development and the ability of immune system against cancer tissues.

Another mechanism causing the different survival outcomes may be that GMFG appears strongly correlated with different immunomodulators including costimulators, co-inhibitors, ligands, receptors, cell adhesion and antigen presentation molecules in different cancers. Meanwhile, the strongest correlations between GMFG and immunomodulators were found in TGCT, SKCM, UVM, LUSC and COADREAD. However, GMFG was negatively correlated with most of the immunomodulators in THYM, and was weakly correlated with the immunomodulators of DLBC, LAML, and LIHC. These findings indicate that GMFG may participate in the regulation of cancer immunology. Recent studies reported that GMFG regulates monocyte migration by modulating ITGB1 [22], and functions as a negative regulator of Toll-like receptor 4 (TLR4) signaling through facilitating TLR4 endocytic trafficking in macrophages [23]. Interestingly, our study also found a strong correlation between GMFG and TLR4 as well as ITGB2. The significant associations between GMFG and immunomodulators show that GMFG modulates the migration, adhesion and activation of immunocytes through regulating immunomodulators, thus functions in the immune response of various cancers. Besides, GMFG is a cytokine-responsive protein mediating the pluripotential and lineage commitment of human hematopoietic stem cells [24], and is downregulated in the process of erythroid maturation and the response to LPS [25]. Apart from immune response, we show that GMFG also participates in cytokine-cytokine receptor interaction and hematopoietic cell lineage pathways, which is consistent with the previous studies. Furthermore, a study [26] reported that GMFG modulates the iron metabolism and M2 polarization of macrophage via inducing mitochondrial ROS and serves as a novel therapeutic target in immune and metabolic disorders. Therefore, GMFG may also act as a regulator of immunomodulators and a cytokine-responsive protein in modulating tumor immunity and hematopoiesis in addition to serving as an
ADF-H protein in remodeling the actin cytoskeleton. GMFG may involve in the cancer immunity and hematopoiesis through interacting with different immunomodulators and cytokines, leading to different immune response status and tumor progression in different types of cancer.

Moreover, we found that GMFG had a significantly positive association with PD-1 and PD-L2, but not with PD-L1 in all cancers except for THYM, DLBC, LAML, and LIHC. Interestingly, the expression of GMFG was also obviously higher in THYM, DLBC and LAML than that in other cancer types, and GMFG was mainly enriched in the plasma membrane in most cancers, but was strongly concentrated on the nucleoplasm or extracellular exosome in THYM, DLBC and LAML. A previous bioinformatics study also demonstrated a weak correlation between PD-1 and other immunomodulators in THYM, LAML, and DLBC, while a strong correlation among them was observed in other types of cancer [27]. These results imply that GMFG has a weak interaction with immunomodulators and a different role in THYM, LAML and DLBC, and these immune and hematopoiesis system-related tumors may have a different immune tumor microenvironment (TME) compared to other solid cancers, which has been proved in recent studies [28-30]. PD-1 is a critical immune checkpoint in TME [31] which functions in adaptive resistance for cancer in immune escape [32]. PD-L1 and PD-L2 are important co-inhibitors for the immune inhibitory impact of PD-1 [33], PD-L1 is expressed in both tumor-infiltrating immune cells and tumor cells [34]. While it was initially suggested that PD-L2 expression is much more restricted than PD-L1 expression, and PD-L2 is expressed mainly in immune cells [35], but recent studies demonstrate that PD-L2 is also expressed in various types of tumor cells, depending on the different TME situations $[36,37]$. What's more, PD-L2 is also found to be significantly associated with the progression of some cancers $[38,39]$. Thus, GMFG may interact with PD-1 and PDL2 to promote the immune escape and progression of cancers.

Our study preliminary identified the potentially functional mechanisms of GMFG including protein binding, immune response and cytokine-cytokine receptor interaction. These findings may help to understand the real mechanisms of GMFG in regulating the tumor progression, immune response status and TME in different cancers. Therefore, in order to fully understand the role of GMFG, future cancer research on GMFG should not only focus on the function of GMFG in modulating of the actin cytoskeleton of cancer cell, cancer-associated vascular endothelial cells or immunocytes, but also pay attention to the cytokine-responsive function and immunomodulation impact of this factor, as well as its role in 
the tumor hematopoiesis and immune TME, especially its impacts on the motility and activation of the tumorinfiltrating immune cells, since the immune composition of the TME including the innate and adaptive immunocytes is a critical determinant of tumor development [40]. Meanwhile, the crosstalk between GMFG and immunomodulators should also be addressed in future studies. What's more, our study also provides the overlapping co-expression genes in the cytokinecytokine receptor interaction pathways, future studies may investigate the interaction between GMFG and these cytokines to better understand the role of GMFG in cancers. However, there are some limitations should be mentioned in this study. First, results of this study were mainly obtained by bioinformatics, the detailed mechanisms have not been verified by experiments. Thus, future experimental research should be performed to prove these pathways. Second, except for pathological stages, we failed to analyze the relationship between GMFG and other clinicopathological parameters of pancancers. In addition, this study mainly discussed GMFGrelated KEGG pathways of cytokine-cytokine receptor interaction, the biological process of immune response, and the molecular function of protein binding, only limited attentions were paid to other GO/KEGG pathways such as cell adhesion molecules, signal transduction and receptor activity, which might also be critical. Finally, we mainly focused on mining and discussing the potential signaling of most cancers since they shared the same GO/KEGG pathways, we did not pay too much attention to the pathways of DLBC, LAML, and LIHC that were different from that of most cancer types.

\section{Conclusion}

Our study demonstrates that the expression of GMFG varies in different cancers, and high expression of GMFG predicts different prognoses for different cancers. GMFG is mainly involved in immune response, protein binding and cytokine-cytokine receptor interaction pathways, and is positively associated with immunomodulators in most cancers. Overall, our findings may help to understand the real mechanisms of GMFG in regulating the tumor progression, immune response status and tissue-specific TME of different cancers.

\footnotetext{
Abbreviations

GMFG: Glia maturation factor $\gamma_{;}$ADF-H: Actin-depolymerizing factor homology; Arps: Actin-related proteins; TCGA: The Cancer Genome Atlas; GO: Gene Ontology; KEGG: Kyoto Encyclopedia of Genes and Genomes; DAVI D: Database for Annotation, Visualization and Integrated Discovery; GBM: Glioblastoma; KIRC: Kidney clear cell carcinoma; LGG: Lower grade glioma; LAML: Acute myeloid leukemia; PAAD: Pancreatic cancer; TGCT: Testicular cancer; KICH: Kidney chromophobe; LUAD: Lung adenocarcinoma; LUSC: Lung squamous cell carcinoma; OS: Overall survival; DFS: Disease-free survival; HR: Hazard ratio; PRAD: Prostate cancer; SKCM: Skin cutaneous melanoma; THYM: Thymoma; CHOL: Bile duct cancer; GEPIA: Gene Expression Profiling Interactive Analysis; GTEx: Genotype-Tissue Expression;
}

MEM: Multi-Experiment Matrix; DLBC: Diffuse large B-cell lymphoma; MESO: Mesothelioma; PCPG: Pheochromocytoma and paraganglioma; SARC: Sarcoma; UVM: Uveal melanoma; BLCA: Bladder cancer; THCA: Thyroid cancer; STAD: Stage in stomach cancer; COADREAD: Colon and rectal cancer; PD-L2: PDCD1LG2; PD-1: PDCD1; LIHC: Liver cancer; TLR4: Toll-like receptor 4; TME: Tumor microenvironment

\section{Supplementary Information}

The online version contains supplementary material available at https://doi. org/10.1186/s12885-021-08163-2.

\section{Additional file 1.}

\section{Acknowledgments}

None.

\section{Authors' contributions}

Aihua Lan and Gong Yang conceived and designed the study, analyzed data, constructed figures and tables, drafted and critically revised the manuscript. Chunxia Ren, Xiaoling Wang and Guoqing Tong contributed to the criticism, revision and discussion of the manuscript. The authors read and approved the final manuscript.

\section{Funding}

This study was supported by the National Natural Science Foundation of China (No. 81772789, 81572553 and 81372797).

\section{Availability of data and materials}

The data of this study are from The Cancer Genome Atlas (https://portal.gdc. cancer.gov/), the GEPIA (http://gepia.cancer-pku.cn/), the MEM (http://biit.cs. ut.ee/mem), and cBioPortal (http://www.cbioportal.org) databases. Datasets generated for this study are included in the manuscript.

\section{Declarations}

Ethics approval and consent to participate

The TCGA and GTEx datasets are publicly available, no ethical approval is required.

\section{Consent for publication \\ Not applicable.}

\section{Competing interests}

The authors have no conflicts of interest or potential conflicts of interest relevant to this article to disclose.

\section{Author details}

${ }^{1}$ Central Laboratory, the Fifth People's Hospital of Shanghai, Fudan University, Shanghai 200240, China. ${ }^{2}$ Center for Reproductive Medicine, Shuguang Hospital Affiliated to Shanghai University of Traditional Chinese Medicine, Shanghai 200120, China. ${ }^{3}$ Cancer Institute, Fudan University Shanghai Cancer Center, Department of Oncology, Fudan University Shanghai Medical College, Shanghai 200032, China.

Received: 3 February 2021 Accepted: 6 April 2021

Published online: 17 April 2021

\section{References}

1. Pettmann B, Sensenbrenner M, Labourdette $G$. Isolation of a glial maturation factor from beef brain. FEBS Lett. 1980;118(2):195-9. https://doi. org/10.1016/0014-5793(80)80217-1.

2. Kaplan R, Zaheer A, Jaye M, Lim R. Molecular cloning and expression of biologically active human glia maturation factor-beta. J Neurochem. 1991; 57(2):483-90. https://doi.org/10.1111/j.1471-4159.1991.tb03777.x.

3. Ikeda K, Kundu RK, Ikeda S, Kobara M, Matsubara H, Quertermous T. Glia maturation factor-gamma is preferentially expressed in microvascular endothelial and inflammatory cells and modulates actin cytoskeleton reorganization. Circ Res. 2006;99(4):424-33. https://doi.org/10.1161/01.RES. 0000237662.23539.0b. 
4. Goroncy AK, Koshiba S, Tochio N, Tomizawa T, Sato M, Inoue M, et al. NMR solution structures of actin depolymerizing factor homology domains. Protein Sci. 2009;18(11):2384-92. https://doi.org/10.1002/pro.248.

5. Goode BL, Sweeney MO, Eskin JA. GMF as an actin network remodeling factor. Trends Cell Biol. 2018;28(9):749-60. https://doi.org/10.1016/j.tcb.2 018.04.008.

6. Tedeschi A, Dupraz S, Curcio M, Laskowski CJ, Schaffran B, Flynn KC, et al. ADF/Cofilin-Mediated Actin Turnover Promotes Axon Regeneration in the Adult CNS. Neuron. 2019;103(6):1073-85 e1076.

7. Zuo P, Fu Z, Tao T, Ye F, Chen L, Wang X, et al. The expression of glia maturation factors and the effect of glia maturation factor- $\gamma$ on angiogenic sprouting in zebrafish. Exp Cell Res. 2013;319(5):707-17. https://doi.org/10.1 016/j.yexcr.2013.01.004

8. Wang T, Cleary RA, Wang R, Tang DD. Glia maturation factor- $\gamma$ phosphorylation at Tyr-104 regulates actin dynamics and contraction in human airway smooth muscle. Am J Respir Cell Mol Biol. 2014;51(5):652-9. https://doi.org/10.1165/rcmb.2014-01250C.

9. Ihrie MD, Ingram JL. Orchestrating airway smooth muscle cell migration: GMFy phosphorylation is the key. Am J Respir Cell Mol Biol. 2019;61(2):1368. https://doi.org/10.1165/rcmb.2019-0074ED.

10. Gerlach BD, Tubbesing K, Liao G, Rezey AC, Wang R, Barroso M, et al. Phosphorylation of GMFY by c-Abl coordinates Lamellipodial and focal adhesion dynamics to regulate airway smooth muscle cell migration. Am J Respir Cell Mol Biol. 2019;61(2):219-31. https://doi.org/10.1165/ rcmb.2018-03520C.

11. Lippert DN, Wilkins JA. Glia maturation factor gamma regulates the migration and adherence of human T lymphocytes. BMC Immunol. 2012; 13(1):21. https://doi.org/10.1186/1471-2172-13-21.

12. Aerbajinai W, Liu L, Chin K, Zhu J, Parent CA, Rodgers GP. Glia maturation factor- $\gamma$ mediates neutrophil chemotaxis. J Leukoc Biol. 2011;90(3):529-38. https://doi.org/10.1189/jlb.0710424.

13. Popinako A, Antonov M, Dibrova D, Chemeris A, Sokolova OS. Analysis of the interactions between GMF and Arp2/3 complex in two binding sites by molecular dynamics simulation. Biochem Biophys Res Commun. 2018;496(2): 529-35. https://doi.org/10.1016/j.bbrc.2018.01.080.

14. Chánez-Paredes S, Montoya-García A, Schnoor M. Cellular and pathophysiological consequences of Arp2/3 complex inhibition: role of inhibitory proteins and pharmacological compounds. Cell Mol Life Sci. 2019; 76(17):3349-61. https://doi.org/10.1007/s00018-019-03128-y.

15. Zuo $P, M a ~ Y$, Huang $Y, Y e F$, Wang $P$, Wang $X$, et al. High GMFG expression correlates with poor prognosis and promotes cell migration and invasion in epithelial ovarian cancer. Gynecol Oncol. 2014;132(3):745-51. https://doi. org/10.1016/j.ygyno.2014.01.044.

16. Wang H, Chen Z, Chang H, Mu X, Deng W, Yuan Z, et al. Expression of glia maturation factor $\gamma$ is associated with colorectal cancer metastasis and its downregulation suppresses colorectal cancer cell migration and invasion in vitro. Oncol Rep. 2017;37(2):929-36. https://doi.org/10.3892/or.2017.5361.

17. Tang Z, Li C, Kang B, Gao G, Li C, Zhang Z. GEPIA: a web server for cancer and normal gene expression profiling and interactive analyses. Nucleic Acids Res. 2017;45(W1):W98-w102. https://doi.org/10.1093/nar/gkx247.

18. GTEx Consortium. Human genomics. The Genotype-Tissue Expression (GTEx) pilot analysis: multitissue gene regulation in humans. Science. 2015; 348(6235):648-60. https://doi.org/10.1126/science.1262110.

19. Adler P, Kolde R, Kull M, Tkachenko A, Peterson H, Reimand J, et al. Mining for coexpression across hundreds of datasets using novel rank aggregation and visualization methods. Genome Biol. 2009;10(12):R139. https://doi.org/1 0.1186/gb-2009-10-12-r139.

20. Kanehisa M, Goto S. KEGG: Kyoto encyclopedia of genes and genomes. Nucleic Acids Res. 2000;28(1):27-30. https://doi.org/10.1093/nar/28.1.27.

21. Thorsson V, Gibbs DL, Brown SD, Wolf D, Bortone DS, Ou Yang TH, et al. The Immune Landscape of Cancer. Immunity. 2018;48(4):812-30 e814.

22. Aerbajinai W, Liu L, Zhu J, Kumkhaek C, Chin K, Rodgers GP. Glia maturation factor-gamma regulates monocyte migration through modulation of beta1integrin. J Biol Chem. 2016;291(16):8549-64. https://doi.org/10.1074/jbc.M11 5.674200 .

23. Aerbajinai W, Lee K, Chin K, Rodgers GP. Glia maturation factor-gamma negatively modulates TLR4 signaling by facilitating TLR4 Endocytic trafficking in macrophages. J Immunol. 2013;190(12):6093-103. https://doi. org/10.4049/jimmunol.1203048.

24. Shi Y, Chen L, Liotta LA, Wan HH, Rodgers GP. Glia maturation factor gamma (GMFG): a cytokine-responsive protein during hematopoietic lineage development and its functional genomics analysis. Genomics Proteomics Bioinformatics. 2006;4(3):145-55. https://doi.org/10.1016/S16720229(06)60027-2.

25. Aerbajinai W, Lee K, Chin K, Rodgers GP. Glia maturation factor- $\gamma$ negatively modulates TLR4 signaling by facilitating TLR4 endocytic trafficking in macrophages. J Immunol. 2013;190(12):6093-103. https://doi.org/10.4049/ jimmunol.1203048.

26. Aerbajinai W, Ghosh MC, Liu J, Kumkhaek C, Zhu J, Chin K, et al. Glia maturation factor- $\gamma$ regulates murine macrophage iron metabolism and $\mathrm{M} 2$ polarization through mitochondrial ROS. Blood Adv. 2019;3(8):1211-25. https://doi.org/10.1182/bloodadvances.2018026070.

27. Shang J, Song Q, Yang Z, Sun X, Xue M, Chen W, et al. Analysis of PD-1 related immune transcriptional profile in different cancer types. Cancer Cell Int. 2018;18(1):218. https://doi.org/10.1186/s12935-018-0712-y.

28. Récher C. Clinical implications of inflammation in acute myeloid leukemia. Front Oncol. 2021;11:623952. https://doi.org/10.3389/fonc.2021.623952.

29. Yamada Y, Weis CA, Thelen J, Sticht C, Schalke B, Ströbel P, et al. Thymoma associated myasthenia gravis (TAMG): differential expression of functional pathways in relation to MG status in different Thymoma Histotypes. Front Immunol. 2020;11:664. https://doi.org/10.3389/fimmu.2020.00664.

30. Scott DW, Gascoyne RD. The tumour microenvironment in B cell lymphomas. Nat Rev Cancer. 2014;14(8):517-34. https://doi.org/10.1038/nrc3774.

31. Xie M, Huang X, Ye X, Qian W. Prognostic and clinicopathological significance of PD-1/PD-L1 expression in the tumor microenvironment and neoplastic cells for lymphoma. Int Immunopharmacol. 2019;77:105999. https://doi.org/10.1016/j.intimp.2019.105999.

32. Zou W, Wolchok JD, Chen L. PD-L1 (B7-H1) and PD-1 pathway blockade for cancer therapy: Mechanisms, response biomarkers, and combinations. Sci Transl Med. 2016;8(328):328rv324.

33. Zak KM, Grudnik P, Magiera K, Dömling A, Dubin G, Holak TA. Structural Biology of the Immune Checkpoint Receptor PD-1 and Its Ligands PD-L1/ PD-L2. Structure. 2017;25(8):1163-74.

34. Garcia-Diaz A, Shin DS, Moreno BH, Saco J, Escuin-Ordinas H, Rodriguez GA, et al. Interferon receptor signaling pathways regulating PD-L1 and PD-L2 expression. Cell Rep. 2017;19(6):1189-201. https://doi.org/10.1016/j.celrep.2 017.04.031.

35. Keir ME, Butte MJ, Freeman GJ, Sharpe AH. PD-1 and its ligands in tolerance and immunity. Annu Rev Immunol. 2008;26(1):677-704. https://doi.org/1 0.1146/annurev.immunol.26.021607.090331.

36. Ohigashi Y, Sho M, Yamada Y, Tsurui Y, Hamada K, Ikeda N, et al. Clinical significance of programmed death-1 ligand-1 and programmed death-1 ligand-2 expression in human esophageal cancer. Clin Cancer Res. 2005; 11(8):2947-53. https://doi.org/10.1158/1078-0432.CCR-04-1469.

37. Yearley JH, Gibson C, Yu N, Moon C, Murphy E, Juco J, et al. PD-L2 expression in human tumors: relevance to anti-PD-1 therapy in Cancer. Clin Cancer Res. 2017;23(12):3158-67. https://doi.org/10.1158/1078-0432.CCR-1 6-1761.

38. Nakayama Y, Mimura K. Immune suppression caused by PD-L2 expression on tumor cells in gastric cancer; 2020.

39. Baptista MZ, Sarian LO, Derchain SF, Pinto GA, Vassallo J. Prognostic significance of PD-L1 and PD-L2 in breast cancer. Hum Pathol. 2016;47(1): 78-84. https://doi.org/10.1016/j.humpath.2015.09.006.

40. Chen H, Chong W, Yang X, Zhang Y, Sang S, Li X, et al. Age-related mutational signature negatively associated with immune activity and survival outcome in triple-negative breast cancer. Oncoimmunology. 2020; 9(1):1788252. https://doi.org/10.1080/2162402X.2020.1788252.

\section{Publisher's Note}

Springer Nature remains neutral with regard to jurisdictional claims in published maps and institutional affiliations. 